\title{
Comparison of Blood Glucose Variability Between Exenatide and Biphasic Insulin Aspart 30 in Chinese Participants with Type 2 Diabetes Inadequately Controlled with Metformin Monotherapy: A Multicenter, Open-Label, Randomized Trial
}

\author{
Li Wang $\cdot$ Xiangyang Liu · Wenjuan Yang · Jingbo Lai · \\ Xinwen $\mathrm{Yu} \cdot \mathrm{Jianrong} \mathrm{Liu} \cdot \mathrm{Xiling}$ Gao $\cdot$ Jie Ming $\cdot$ Kaiyan Ma • \\ Jing $\mathrm{Xu} \cdot$ Zhufang Tian $\cdot$ Qingzhen He $\cdot$ Qiuhe Ji \\ Received: June 2, 2020 / Published online: August 27, 2020 \\ (C) The Author(s) 2020
}

\section{ABSTRACT}

Introduction: To compare blood glucose variability (GV) in Chinese participants with type 2 diabetes mellitus (T2DM) whose blood glucose levels are inadequately controlled with metformin monotherapy after twice-daily exenatide or biphasic insulin aspart 30 (BIAsp30).

Methods: In this 16-week multicenter, randomized clinical trial, 104 participants were randomized 1:1 to receive exenatide (exenatide

Digital Features To view digital features for this article go to https://doi.org/10.6084/m9.figshare.12765704.

Electronic supplementary material The online version of this article (https://doi.org/10.1007/s13300020-00904-z) contains supplementary material, which is available to authorized users.

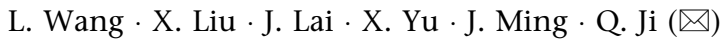
Department of Endocrinology, Xijing Hospital, Fourth Military Medical University, Xi'an, China e-mail: qiuheji@hotmail.com; jqiuhe@fmmu.edu.cn

W. Yang

Department of Endocrinology, Shaanxi Aerospace

Hospital, Xi'an, Shaanxi, China

J. Liu

Department of Endocrinology, Xi'an Chang an

Hospital, Xi'an, Shaanxi, China

X. Gao

Department of Endocrinology, Yan'an People's

Hospital, Yan'an, Shaanxi, China group) or BIAsp30 (BIAsp30 group) twice daily. All participants continued metformin treatment. The primary outcome was the change in $\mathrm{GV}$ as measured by a continuous glucose monitoring system (CGMS) from baseline to 16 weeks.

Results: At 16 weeks, both the Exenatide and BIAsp30 groups effectively decreased mean glucose (MG), but neither group changed the mean amplitude of glycemic excursion (MAGE), largest amplitude of glycemic excursion (LAGE), mean of daily difference (MODD), or standard deviation of blood glucose (SDBG). The decrease in 2-h post-breakfast glucose excursions was greater in the Exenatide group compared to the BIAsp30 group, with a least square (LS) mean difference $[95 \% \mathrm{CI}]$ of $(1.58$ [0.53, 2.63]). Exenatide also significantly reduced 2 -h post-lunch

K. Ma

Department of Endocrinology, Shangluo Central Hospital, Shangluo, Shaanxi, China

J. Xu

Department of Endocrinology, Second Affiliated Hospital of Xi'an Jiaotong University, Xi'an, Shaanxi, China

\section{Z. Tian}

Department of Endocrinology, Xi'an Central

Hospital, Xi'an, Shaanxi, China

Q. He

Department of Endocrinology, Xi'an Gaoxin Hospital, Xi'an, Shaanxi, China 
glucose excursion compared to BIAsp30 (LS mean difference [95\% CI], 1.19 [0.18, 2.20]). The Exenatide group had significantly reduced body weight and body mass index (BMI), while the BIAsp30 group had increased weight and had no change in BMI. Both treatments were well tolerated with no serious hypoglycemic events and with fewer identified hypoglycemic events in the Exenatide group than in the BIAsp30 group $(5.77 \%$ vs. $17.31 \%, P<0.01)$.

Conclusion: Although there was no difference in change of GV between Exenatide and BIAsp30, exenatide provided more improvement in postprandial glucose excursion and weight control, without increasing the risk of hypoglycemia in Chinese patients with T2DM whose blood glucose was inadequately controlled with metformin. These findings may provide new options for patients who choose further hypoglycemic treatment, especially in patients with obesity who have large postprandial plasma glucose excursions.

Trial Registration: ClinicalTrials.gov indentifier: NCT02449603.

Keywords: Biphasic insulin aspart 30 (BIAsp30); Continuous glucose monitoring system (CGMS); Exenatide; Glucose variability; Postprandial glucose excursion

\section{Key Summary Points}

\section{Why carry out this study?}

This study showed exenatide treatment resulted in $\mathrm{HbA1c}$ reduction similar to that of BIAsp30. However, HbA1c level does not reflect $\mathrm{GV}$, and there has been accumulating evidence supporting the role of GV in the pathogenesis of T2DMassociated vascular complications, such as atherosclerosis and cardiovascular events

Using a CGMS, this study compared differences in GV in Chinese participants taking twice-daily exenatide or BIAsp30 whose T2DM was inadequately controlled with metformin monotherapy, which provided beneficial evidence for the use of exenatide in patients with T2DM

\section{What was learned from the study?}

Exenatide provided more improvement in postprandial glucose excursion, weight control, and attenuation of some oxidative stress and inflammatory markers without increased risk of hypoglycemia in Chinese patients with T2DM whose blood glucose was inadequately controlled with metformin

There was no difference in the change for the mean MAGE, LAGE, MODD, and SDBG between the Exenatide and BIAsp30 groups. Exenatide provided greater improvement in postprandial glucose excursion, weight control, and effective control of HbA1c without increasing hypoglycemic events. At the same time, the dosage of exenatide did not need to be titrated to blood glucose levels like the dosage of BIAsp30 requires. The results of this study provide new evidence to support the use of novel options for patients who choose insulin to further hypoglycemic treatment, especially in patients with obesity

\section{INTRODUCTION}

An estimated $70 \%$ of Chinese patients with type 2 diabetes mellitus (T2DM) who are initiating insulin therapy use premixed insulin [1]. BIAsp30 is a premixed insulin formulation designed to provide both basal and postprandial glycemic control by retaining the fast onset of action associated with insulin aspart and extending its duration of action [2]. Because of the cost-effective advantage in the management of diabetic complications and hypoglycemia, BIAsp30 has been used by millions of patients, and its effectiveness and safety have been widely recognized since 2002 [3, 4]. However, there are some unavoidable side effects of insulin, including increased risk of hypoglycemia, weight gain, and edema. At the same 
time, patients must perform a complex insulin titration scheme to regulate their blood glucose [5-7].

Exenatide belongs to the glucagon-like peptide-1 receptor agonist (GLP-1RA) class of medications [8], which enhances glucose-dependent insulin secretion, suppresses abnormally increased postprandial glucagon secretion, reduces food intake, and slows down gastric emptying $[9,10]$. Exenatide can reduce glycated hemoglobin (HbA1c) as well as postprandial blood glucose, while effectively reducing body weight in patients with T2DM $[8,11-15]$. Unlike insulin, exenatide dosing does not need to be titrated to blood glucose levels. When combined with metformin, exenatide does not increase the risk of hypoglycemia $[13,16]$.

In 2020, ESC/EASD improved the recommendation level of GLP-1RA use, indicating that the use of GLP-1RA is preferred in patients with atherosclerotic cardiovascular disease (ASCVD) or those who are high/very high risk [17]. The latest ADA guidelines also suggest that the choice of medication added to metformin should be based on the clinical characteristics of the patients and their preferences. In patients with established ASCVD or indicators of high ASCVD risk, a GLP-1RA or a GLT-2 inhibitor with demonstrated CVD benefit is recommended as part of the glucose-lowering regimen independent of HbA1c [18].

A randomized, controlled study (RCT) of exenatide and BIAsp30 demonstrated treatment with exenatide resulted in $\mathrm{HbA1c}$ reduction similar to that of BIAsp30 and provided better postprandial glycemic control [19]. Proper glycemic control achieved by lowering HbA1c can reduce vascular complications associated with T2DM [20]. However, HbA1c level does not reflect glucose variability $(\mathrm{GV})$, which has been implicated in the pathogenesis of T2DM-associated vascular complications, such as atherosclerosis and cardiovascular (CV) events [21-23]. GV contributes to T2DM-associated vascular complications by activating oxidative stress and inflammatory reactions [24, 25]. Some inflammatory factors, such as 8-iso-PGF2 $\alpha(8$-iso-PGF2 $\alpha)$ and MCP-1, play an important role in predicting the risk of cardiovascular eventsin diabetes patients $[26,27]$.
Large serum glucose fluctuations may have more deleterious effects than continuous hyperglycemia and can be an independent risk factor for diabetes-associated CV complications $[28,29]$. Postprandial GV is one parameter of GV. Both postprandial GV and especially postprandial hyperglycemia have been shown to contribute to late diabetic complications and the incidence of CV events and mortality in the general population [30]. High GV also increases the risk of hypoglycemia, and hypoglycemia is associated with diabetes-related vascular complications by stimulating oxidative stress, inflammatory reactions, and endothelial abnormalities. Therefore, GV is becoming an important concern in diabetes control [21].

Use of a continuous glucose monitoring system (CGMS) provides a precise and reliable method for evaluating GV as it continuously measures glucose levels every $5 \mathrm{~min}$. Using CGMS, the primary aim of this study was to compare exenatide's efficacy in GV control versus BIAsp30 in Chinese patients with T2DM whose blood glucose was inadequately controlled with metformin monotherapy. We hope the results of this study can provide a basis for further optimization of current diabetes treatment strategies.

\section{METHODS}

\section{Study Design and Ethics Compliance}

Details of the study design and methods were previously published [1]. This multicenter, randomized, open-label trial was planned to be conducted in eight study sites in China and consisted of a 1-week screening period and a 16-week treatment period. Six study sites participated in the trial. This study was designed and conducted per the Good Clinical Practice (GCP), the Chinese GCP. This trial was approved by the Independent Ethics Committee of Xijing Hospital, First Affiliated Hospital of Fourth Military Medical University (ID: KY20150319, KY20150505-1) (Xi'an, China), the Second Affiliated Hospital of Xi'an Jiaotong University (Xi'an, China), Chang An Hospital (Xi'an, China), Xi'an Gaoxin Hospital (Xi'an, 
China), Yan'an People's Hospital (Yan'an), and China and Shangluo Central Hospital (Shangluo, China), and written informed consent was obtained from all participants. This trial was performed as per the ethics delineated in the Helsinki Declaration. This study is registered with ClinicalTrials.gov: NCT02449603.

\section{Participants}

Participants who fulfilled the following criteria were included: men and non-pregnant women (using a medically approved birth-control method) between 18 and 70 years old at screening, with confirmed T2DM $\geq 6$ months before the study, treatment with metformin at a stable, maximally tolerated dose ( $\geq 1500 \mathrm{mg} /$ day) for $\geq 3$ months, with HbA1c $\geq 7.5 \%$ and $\leq 10.0 \%$ at screening or within 4 weeks before the screening, and with a body mass index (BMI) of $21-35 \mathrm{~kg} / \mathrm{m}^{2}$. Exclusion criteria are listed in Appendix S1.

\section{Intervention}

All eligible participants were randomized 1:1 to one of the two treatments (exenatide or BIAsp30 insulin) through a central randomization system using an interactive voice/web response system. This was an open-label study; however, investigators were blinded to data on key efficacy parameters such as the mean amplitude of glycemic excursion (MAGE), largest amplitude of glycemic excursions (LAGE), mean of daily difference (MODD), standard deviation of blood glucose (SDBG), mean glucose (MG), postprandial glucose excursion, HbA1c, fasting plasma glucose (FPG), and postprandial plasma glucose (PPG) of standard meal testing.

The study included a 1-week screening period during which participants received $1500 \mathrm{mg}$ /day metformin and a 16-week treatment period during which the participants took oral background metformin $(500 \mathrm{mg}, 3$ times a day) and one of the study drugs.

Participants in the Exenatide group received $5 \mu \mathrm{g}$ exenatide (AstraZeneca, London, UK) through each subcutaneous injection, twice a day (bid), $60 \mathrm{~min}$ before breakfast, and $60 \mathrm{~min}$ before dinner, during the first 4 weeks of the treatment, and then they received $10 \mu \mathrm{g}$ exenatide bid during the next 12 weeks. Participants in the BIAsp30 group received BIAsp30 insulin (Novo Nordisk, Bagsværd, Denmark) through subcutaneous injection starting at a dose of $0.2-0.4 \mathrm{IU} / \mathrm{kg}$ or $10-12 \mathrm{IU} /$ day, split into two equal doses before breakfast and before dinner. Doses of BIAsp 30 were titrated to a target pre-breakfast fasting glucose and predinner glucose of $5.0-7.0 \mathrm{mmol} / \mathrm{l}$ based on selfmeasured blood glucose (SMBG) values 3 days before the visit (Appendix S2). Investigators provided BIAsp 30 dosing recommendations according to titration guidelines used in previous treat-to-target studies [5]. Insulin dose adjustment was conducted on an individual basis to achieve an optimal balance between proper glycemic control (target FPG and predinner plasma glucose $<7 \mathrm{mmol} / \mathrm{l}$ ) and to minimize the risk of hypoglycemia as dictated by clinical practice. Detailed insulin dose titration was performed according to Xu et al. [1].

\section{Outcomes}

The primary efficacy outcome was the absolute change of GV at week 16 from baseline, including MAGE, LAGE, SDBG, MODD, and MG. Secondary efficacy outcomes included changes from baseline to week 16 in 2-h PPG excursion, HbA1c, FPG of the standard meal, 2-h PPG of the standard meal, body weight, BMI, 8-iso-prostaglandin $F_{2 \alpha}$ (8-iso-PGF2 $\alpha$ ), monocyte chemotactic protein-1 (MCP-1), and hypoglycemic events.

Blood glucose was monitored at each site by CGMS (Guardian ${ }^{\mathrm{TM}}$ Real-Time CGMS, Medtronic MiniMed Inc., Northridge, CA, USA), which recorded glucose levels every $5 \mathrm{~min}$ for 72 consecutive hours. The attachment of the device onto the participants and its operation, calibration, and data collection were conducted according to $\mathrm{Xu}$ et al.'s report [1]. MAGE, MG, LAGE, SDBG, and MODD were calculated from data extracted from each CGMS output using the CGMS 3.0 software package (Medtronic MiniMed, MMT-7310 version 3.0C [3.0.128], 
CA, USA), whereas FPG and 2-h PPG were measured from the standard meal. The 2-h PPG excursion was calculated as the difference between 2-h post-breakfast/lunch/dinner plasma glucose and pre-breakfast/lunch/dinner plasma glucose measured by SMBG levels.

Plasma samples isolated from venous blood samples after overnight fasting collected at baseline and week 16 were stored at $-80^{\circ} \mathrm{C}$. MCP-1 and PGF2a levels were measured with enzyme-linked immunosorbent assay kits (Shanghai Chang Jin Biotechnology Co., Ltd., Shanghai, China), whereas Hs-CRP was determined with radioimmunoassay kits (Siemens, Marburg, Germany).

To assess safety and tolerability of the two drugs, adverse events (AEs) including reported hypoglycemia (patient-reported symptoms of hypoglycemia that promptly resolved after food intake or intravenous glucose), identified hypoglycemia (a blood glucose level $\leq 3.9 \mathrm{mmol} / 1$ regardless whether there were symptoms of hypoglycemia), severe hypoglycemia (the patient needed assistance from another person due to severely impaired consciousness or behavior), serious adverse events (SAEs), physical examinations including vital signs, clinical hypoglycemia, electrocardiogram, routine laboratory chemistry, and hematologic tests were performed using standard methods.

\section{Statistical Analyses}

One hundred fifty participants were each randomized 1:1 to one of the two treatment groups for this trial. This sample size was expected to provide at least $85 \%$ power to test a minimum $1.6 \mathrm{mmol} / \mathrm{l}$ difference on the change of MAGE, the primary end point, between the Exenatide and BIAsp30 groups (two-sided $\alpha=0.05$, estimated $15 \%$ dropout rate, estimated SD of change of MAGE $\approx 3.0 \mathrm{mmol} / \mathrm{l}$ ) [1]. However, we ran into some unexpected difficulties in recruiting participants for our study. To prevent potentially endless delays, we decided to conduct the study on the 104 participants we had already enrolled at that time.
Efficacy analyses were conducted on the full analysis set (FAS), comprising all randomized participants who received $\geq 1$ dose of study drug and had at least one follow-up visit, with the last observation carried forward (LOCF). The per-protocol set (PPS) comprising all randomized participants who completed the study with no major protocol violation that affected study outcomes was used for supportive analyses. Safety analyses were conducted on the safety set (SS) of all randomized participants who received $\geq 1$ dose of study drug and who had baseline safety data and safety data for at least one follow-up visit.

SPSS 19.0 software (SPSS Inc., Chicago, IL, USA) was used to perform all statistical analyses. Data were presented as mean \pm standard deviation (SD), median (minimum, maximum), or a percentage. Generally, to evaluate withingroups differences, the Student's $t$ test or MannWhitney $U$ test was used for continuous variables, while the chi-square test was used for categorical variables. Differences in efficacy parameters within and between the two treatment groups over time were evaluated using analysis of covariance (ANCOVA) with baseline values as covariate and treatment site effect as the fixed factor and presented as least square (LS) means with corresponding Dunnett 95\% confidence interval (95\% CI). Dunnett LS mean differences $(95 \% \mathrm{CI})$ were provided for differences between the groups. Differences were considered to be statistically significant when the $95 \%$ CI did not include 0 . Incidence rates of adverse events of the two groups were compared with the Fisher's exact test. Statistical significance was accepted with $P<0.05$.

\section{RESULTS}

\section{Participants Demographics and Baseline Characteristics}

Of the 134 participants screened, 104 participants were randomized 1:1 to receive exenatide (the Exenatide group) or BIAsp30 (BIAsp30 group). Twelve participants in the Exenatide group and four in the BIAsp30 group withdrew from the study. A final 88 (84.6\%) participants 
completed the study. The FAS included 104 participants (52 in each group), the PPS included 88 participants ( 40 and 48 in the Exenatide and the BIAsp30 group, respectively) (Fig. 1), and the SS included 104 participants (52 in each group).

Overall, $68 \%$ of the participants were male, and their mean age was 51 years. The mean weight was $74.09 \mathrm{~kg}$, body mass index (BMI) was 26.27, mean T2DM duration was 5.96 years, and mean $\mathrm{HbA} 1 \mathrm{c}$ was $8.75 \%$ in the Exenatide group. The mean weight was $73.56 \mathrm{~kg}$, BMI was 26.25, mean T2DM duration was 6.09 years, and mean HbA1c was $8.81 \%$ in the BIAsp30 group. There were no significant differences in demographics and baseline characteristics between the two groups (Table 1).

\section{Changes of GV Indices as Measured by CGMS}

At 16 weeks, the Exenatide group had a statistically significant decrease in MG versus baseline (LS mean of -0.99 , 95\% CI [- 1.77, $-0.22]$ ), and the BIAsp30 group also had a commensurate decrease in MG versus baseline (LS mean of $-1.37,95 \%$ CI [-2.12, -0.62$]$ ). However, neither treatment significantly reduced LAGE, MAGE, MODD, or SDBG (Table 2).

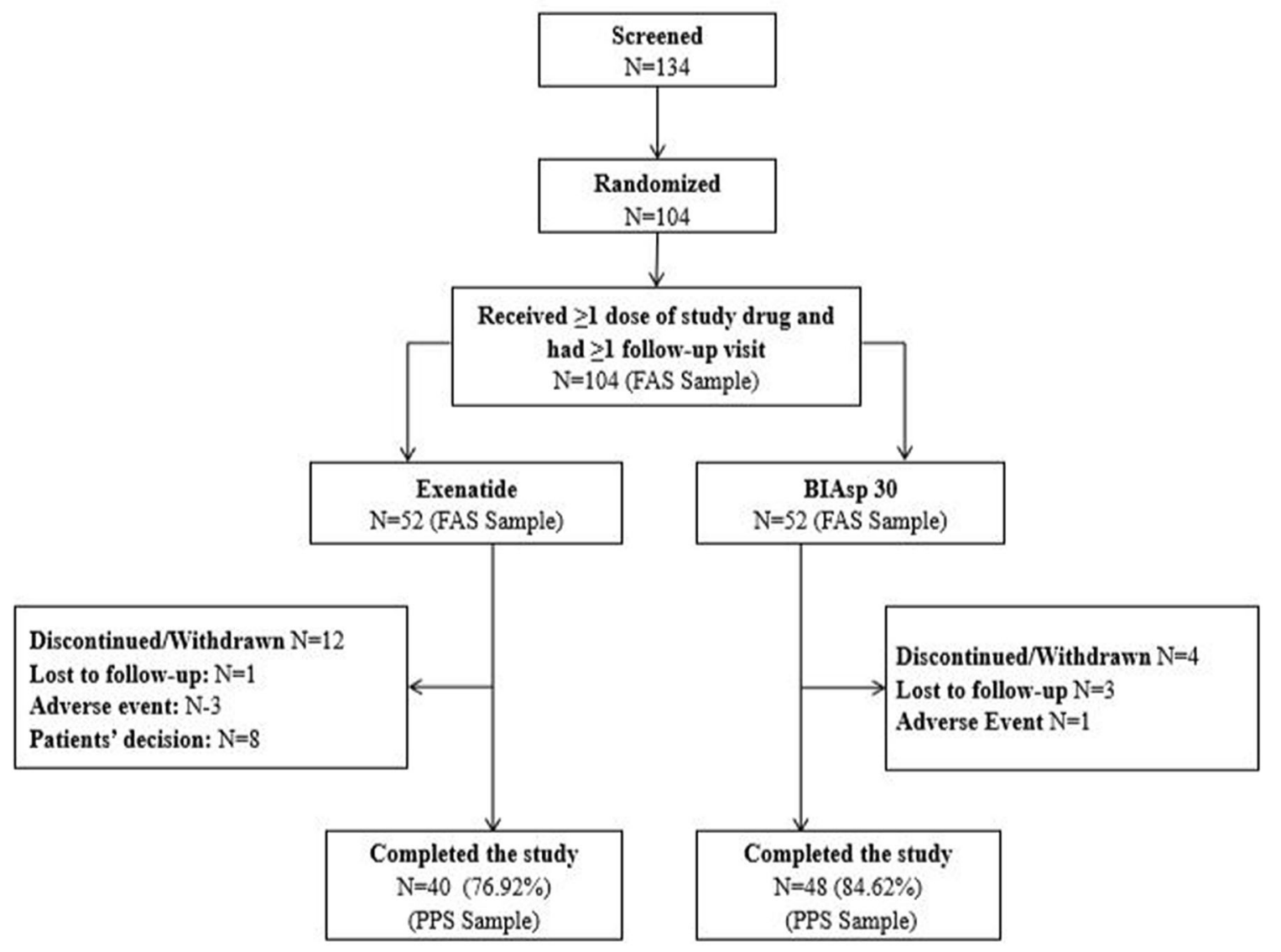

Fig. 1 Flow of participants through the study. FAS full analysis set included all randomized participants receiving at least one dose of any of the trial products. $P A S$ per protocol set, the data set was determined during a blind audit after the completion of the trial, including all cases

that met the trial protocol, had good compliance, had not taken the prohibited drugs during the trial, and had completed the CRF requirements. PPS per protocol set, the population was the secondary population for efficacy evaluation in this study 
Table 1 Patient demographics and baseline characteristics (FAS LOCF)

\begin{tabular}{llll}
\hline Variable & Exenatide $(\boldsymbol{N}=\mathbf{5 2})$ & BIAsp 30 $(\boldsymbol{N}=\mathbf{5 2})$ & $\boldsymbol{P}$ value \\
\hline Male, $n$ (\%) & $36(69.23)$ & $35(67.37)$ & 0.8331 \\
Age, years & $51.54 \pm 9.85$ & $51.61 \pm 9.77$ & 0.9698 \\
Weight, $\mathrm{kg}$ & $74.09 \pm 11.22$ & $73.56 \pm 12.00$ & 0.8162 \\
Height, cm & $167.63 \pm 7.55$ & $167.11 \pm 8.45$ & 0.7399 \\
BMI, $\mathrm{kg} / \mathrm{m}^{2}$ & $26.27 \pm 3.08$ & $26.25 \pm 3.12$ & 0.9691 \\
Duration of T2DM, years & $5.96 \pm 4.15$ & $6.09 \pm 4.46$ & 0.9689 \\
Heart rate, beats/min & $76.35 \pm 6.67$ & $76.06 \pm 6.28$ & 0.8207 \\
SBP, mmHg & $123.27 \pm 10.81$ & $123.00 \pm 12.34$ & 0.906 \\
DBP, mmHg & $79.12 \pm 8.02$ & $76.83 \pm 7.51$ & 0.1361 \\
HbAlc, \% & $8.75 \pm 0.74$ & $8.81 \pm 0.74$ & 0.6422 \\
FPG, mmol/l & $9.96 \pm 2.78$ & $10.26 \pm 2.76$ & 0.574 \\
2-h PPG, mmol/l & $15.92 \pm 3.90$ & $15.89 \pm 2.87$ & 0.3627 \\
Urinary albumin, mg/l & $11.9(2.1-1110)$ & $18.4(2.1-2930)$ & 0.5084 \\
\hline
\end{tabular}

Data were presented as $n$ (\%), mean \pm SD or median (minimum, maximum) in patients who received $\geq 1$ dose of study drug and had at least one follow-up visit

$F A S$ full analysis set, $L O C F$ last observation carried forward, $B M I$ body mass index, $T 2 D M$ types 2 diabetes mellitus, $S B P$ systolic blood pressure, $D B P$ diastolic blood pressure, $H b A 1 c$ glycated hemoglobin, $F P G$ fasting plasma glucose, 2- $h P P G$ 2-h postprandial plasma glucose

The parameters of GV in the two groups were compared (Table 2). There were no differences between the two groups in the changes of GV of MG, MAGE, LAGE, MODD, or SDBG at 16 weeks. The change in the 2 -h post-breakfast glucose excursion was lower in the Exenatide group than in the BIAsp30 group (LS means of $-1.79,95 \%$ CI $[-2.82,-0.75]$ versus -0.20 $[-1.11,0.70])$, with an LS mean difference of $1.58,95 \%$ CI $[0.53,2.63]$, and changes in 2-h post-lunch glucose excursion were also betterthan those seen in the BIAsp30 group (LS means $[95 \% \mathrm{CI}]$ of $-0.53[-1.55,0.48]$ versus $0.65[-0.22,1.53]$, with an LS mean difference of $1.19,95 \%$ CI $[0.18,2.20])$.

There were no differences in changes in 2-h post-dinner glucose excursion between groups.

\section{Changes in Blood Glucose, Hba1c, Body Weight, 8-iso-PGF2 $\alpha$, and MCP-1}

Both the Exenatide and BIAsp30 groups had significantly decreased $\mathrm{HbA1c}$ at 16 weeks versus baseline (LS means [95\% CI] of $-1.38 \%$ $[-1.89,-0.86]$ and $-1.74 \%[-2.20,-1.28]$, respectively) with comparable efficacies (LS mean difference $[95 \% \mathrm{CI}]$ of $-0.36 \%$ [ -0.87 , 0.14]) (Fig. 2a). Participants in both groups showed comparable efficacy in significantly lowering FPG and 2-h PPG measured by standard meal at week 16 versus baseline. The decrease of FPG was not significant between the two groups (LS means $[95 \%$ CI] of -0.81 $[-1.51,-0.12]$ and $-0.92[-1.55,-0.29]$, respectively) with comparable efficacies (LS mean difference $[95 \% \mathrm{CI}]$ of $-0.11,[-0.80$, 0.58]) (Fig. 2b). There was also no difference between groups in the decrease of 2-h PBG (LS 


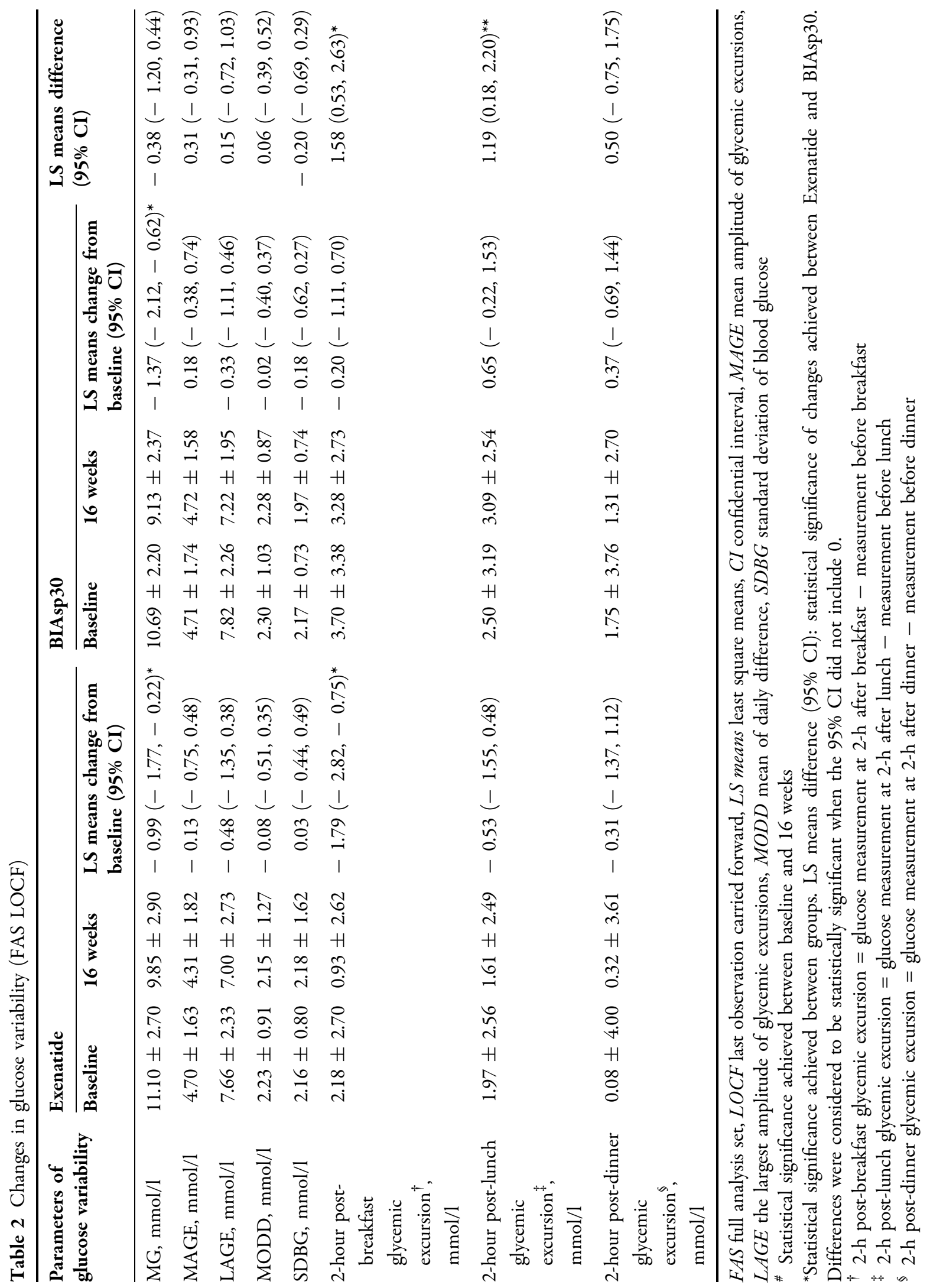


means $[95 \% \mathrm{CI}]$ of $-2.33[-4.14,-0.53]$ and $-1.97[-3.59,-0.36]$, respectively) with comparable efficacies (LS mean difference [95\% $\mathrm{CI}]$ of 0.36 [- 1.43, 2.15]) (Fig. 2c). Importantly, compared to baseline, participants in the Exenatide group had significantly decreased body weight (LS mean $[95 \% \mathrm{CI}]$ of -2.38 [- 3.34, - 1.41]), while participants in the BIAsp30 group had significantly increased body weight (LS mean $[95 \% \mathrm{CI}]$ of $0.89[0.02,1.76])$, and the differences in change of body weight were significant (LS mean difference of 3.27 [2.32, 4.22] kg) (Fig. 2d). Participants in the Exenatide group also had a decrease in BMI (LS mean of -0.88 $[-1.24,-0.53])$, but those in the BIAsp30 group did not, and the differences in change in BMI were significant (LS mean difference of 1.13 $[0.78,1.48])$. There was no difference in reported hypoglycemic events between the two groups; however, the incidence of identified hypoglycemic events in the Exenatide group was lower than that in the BIAsp30 group $(P<0.01)$ (Fig. 2e). There was no significant change in heart rate in the two groups at week 16 versus baseline (Fig. 2f).

At 16 weeks, participants in both the Exenatide and BIAsp30 groups had significantly reduced serum levels of 8 -iso-PGF $2 \alpha$, a marker for oxidative stress (LS means [95\% CI] of $-20.27[-30.20,-10.34]$ and -22.67 [-30.98, - 14.36], respectively), with comparable efficacies (LS mean difference [95\% CI] of -2.41 [- 12.38, 7.56]) (Fig. 2g). A comparably significant decrease in MCP-1, an inflammatory marker, was also observed in the two treatment groups at 16 weeks (LS means [95\% CI] of $-77.09[-94.15,-60.02]$ and -84.59 [-99.15, - 70.02], respectively), with comparable efficacies (LS mean difference [95\% CI] of -7.50 [- 25.04, 10.04]) (Fig. 2h). There was no significant decreased change in 8-iso-PGF2 $\alpha$ and MCP-1 in the two groups.

\section{Safety and Tolerability}

Both treatments were well tolerated. There were 114 incidences of AEs in 37 (71.15\%) participants in the Exenatide group and 105 incidences of AEs in 36 (69.23\%) participants in the
BIAsp30 group, and among them, 71 AEs in 27 participants in the Exenatide group and 56 AEs in 25 participants in the BIAsp30 group were study drug-related. Five severe AEs (SAEs) occurred in four participants in the Exenatide group and five SAEs occurred in three participants in the BIAsp30 group. Three participants in the Exenatide group and one participant in the BIAsp30 group withdrew from the study because of AEs (Fig. 1). Overall, the two treatments had a comparable incidence of AEs (Table 3). Physical examinations including vital signs, electrocardiogram, routine laboratory chemistry, and hematologic tests did not reveal any clinically significant changes for either group.

\section{DISCUSSION}

This RCT aimed to compare GV control efficacy of exenatide versus BIAsp30 in Chinese patients with T2DM whose blood glucose levels were inadequately controlled with metformin monotherapy. We found that both exenatide and BIAsp30 effectively improved MG control. However, neither medication was effective in achieving overall GV control. Exenatide demonstrated better postprandial glucose excursion control than BIAsp30. As expected, both exenatide and BIAsp30 were effective in reducing HbA1c, FPG, and 2-h PPG and had comparable AEs. In addition, exenatide use led to reduced body weight, whereas BIAsp30 use led to weight gain. Neither group experienced serious hypoglycemia, and exenatide had lower identified hypoglycemic events compared to BIAsp30. Our findings are consistent with previous studies on the same topic [31-33] and thus confirm the efficacy of exenatide in general glycemic control.

The primary aim of the current 16-week RCT was to compare exenatide's efficacy in GV control versus BIAsp30. Our results showed that exenatide did not lead to significant changes in MAGE, LAGE, SDBG, or MODD, which is inconsistent with the results of Irace et al. [34]. They conducted a 16-week study including six participants with T2DM who were prescribed exenatide as an adjunct to metformin and 


$$
\text { - Exenatide }
$$

- BIAsp 30

A

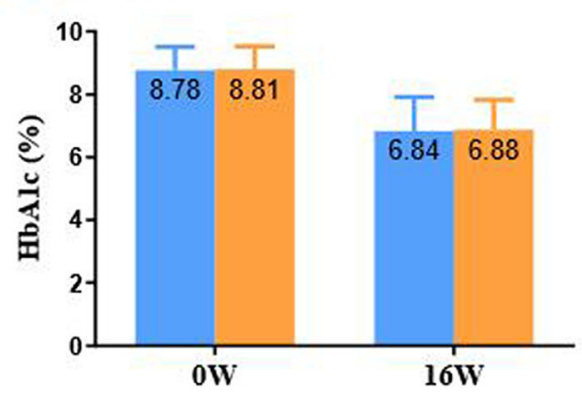

C

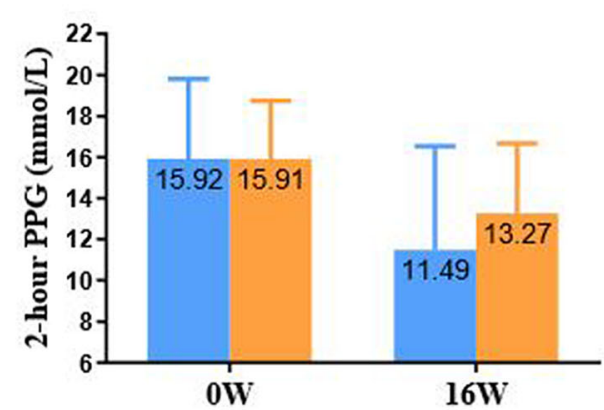

$\mathbf{E}$

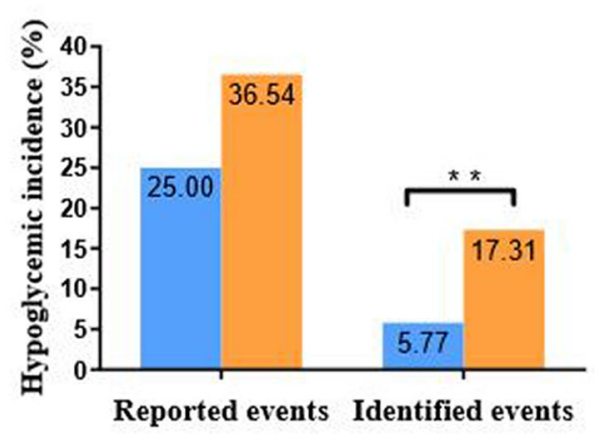

G

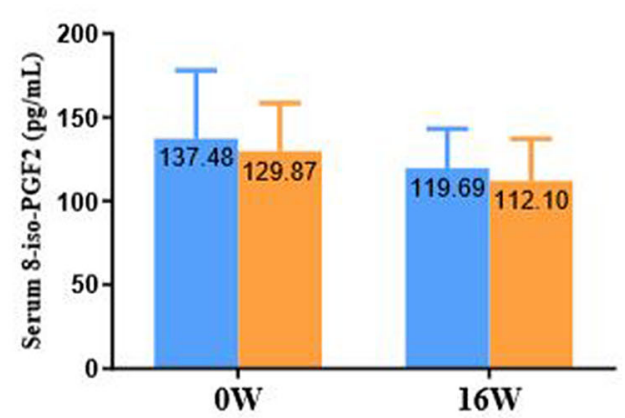

B

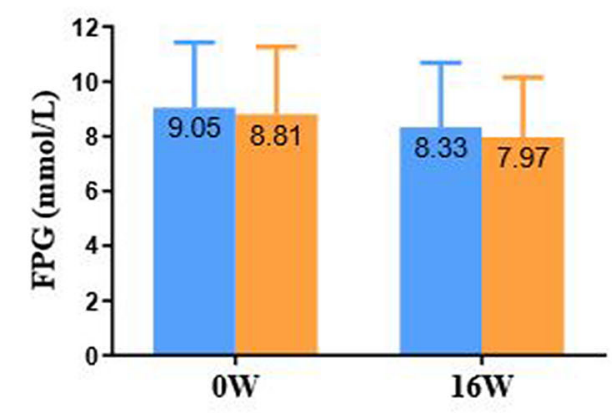

D

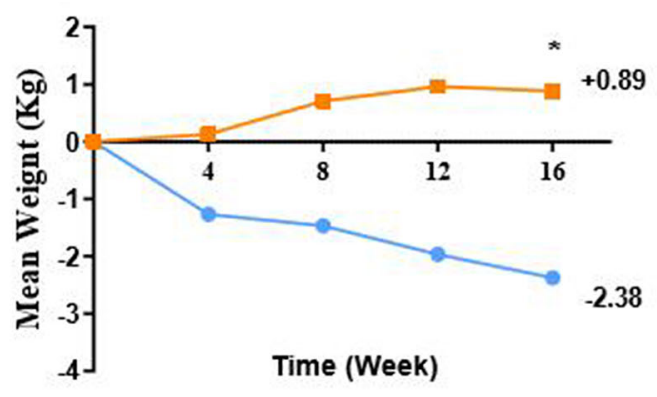

F

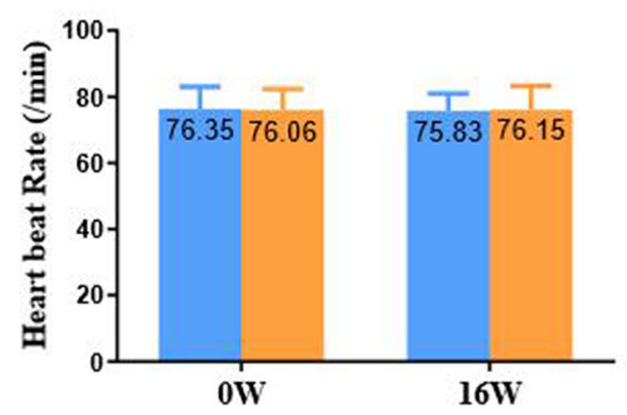

H

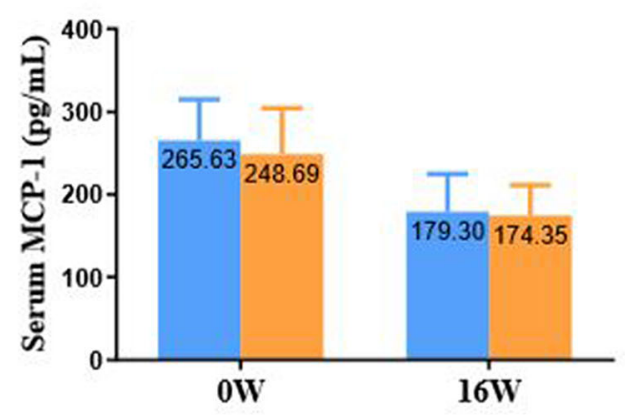


4Fig. 2 Efficacy comparison between Exenatide and BIAsp30 at week 16 (FAS LOCF). a Glycated hemoglobin (HbAlc) values at baseline and week 16. b Fasting plasma glucose (FPG) values at baseline and week 16. c 2-h postprandial plasma glucose values (2-h PPG) at baseline and week 16. d Weight over time, mean of actual value, SD not shown. ${ }^{*} P<0.05$. e Hypoglycemic incidence. Total events, blood glucose level $\leq 3.9 \mathrm{mmol} / \mathrm{l}$; severe events, hypoglycemic events requiring help from others. ${ }^{* *} P<0.01$. f Heart rate at baseline and week 16. g Serum 8 -iso-PGF2 $\alpha$ at baseline and week 16. h Serum MCP-1 at baseline and week 16

found that exenatide significantly decreased MAGE and SDBG. Because of the small sample size, their results should be interpreted with caution. Studies on exenatide as an adjuvant to other hypoglycemic agents have generated inconsistent results regarding whether exenatide could reduce $\mathrm{GV}$ parameters such as MAGE and SDBG [25, 35-37]. Therefore, the ability of exenatide to effectively control overall $\mathrm{GV}$ is less certain at this point. Our study also found that BIAsp30 lacked efficacy in general GV control, a finding inconsistent with two previous studies, which found that BIAsp30 reduced GV [38, 39]. Those two studies had small sample sizes; as such, whether BIAsp30 is efficacious in GV control remains unsettled.

An important finding of our study is that exenatide reduced postprandial glucose excursion, which is consistent with previous studies $[19,25]$. Unlike previous studies $[19,25]$, our study found that exenatide did not change postdinner glucose excursion, possibly because the mean baseline post-dinner glucose excursion in our study was very small $(0.21 \mathrm{mmol} / \mathrm{l})$, much smaller than the mean baseline post-breakfast glucose excursion $(2.35 \mathrm{mmol} / \mathrm{l})$, and also much smaller than that observed in the other two studies of exenatide. Meanwhile, seven-point SMBG profiles have shown that mean blood glucose levels at $2 \mathrm{~h}$ after breakfast and dinner were lower in those taking exenatide than in those taking BIAsp30 at 16 weeks. Because BIAsp30 has superior postprandial glucose excursion control compared to other insulin preparations such as biphasic insulin lispro or biphasic human insulin [2, 19], our findings suggest that exenatide is more efficacious in controlling postprandial glucose excursion than most insulin formulations.

Compared to chronic hyperglycemia, acute glucose excursion and postprandial glucose excursion can lead to increased oxidative stress and increased levels of inflammatory markers $[40,41]$. Reducing postprandial glucose excursion is now considered by some to be important in preventing $\mathrm{CV}$ diseases in patients with diabetes [28]. In combination with previous reports, our findings demonstrate that compared to BIAsp30, exenatide has better efficacy in reducing postprandial GV and lowering the incidence of hypoglycemic events and especially nocturnal hypoglycemic events $[15,42,43]$ and thus has a beneficial and favorable mode of action in diabetes control.

We found that exenatide provided efficacy in glycemic control and a decrease of 8-iso-PGF2 $\alpha$ and MCP-1 comparable to BIAsp30. Patients with diabetes exhibit increased oxidative stress, and the decrease of 8-iso- PGF2 $\alpha$ and MCP-1 suggests both treatments could reduce oxidative stress and inflammatory state. Participants taking exenatide had superior control of both postprandial glucose excursion and body weight. These results suggest exenatide is a valuable and worthy alternative treatment to insulin therapy, considering the $\mathrm{CV}$ benefits associated with weight loss, PPG control, and attenuated oxidative stress and inflammatory conditions [44-47] and the fact that BIAsp30 is already superior to some other insulin formulations in postprandial glucose excursion control [2, 19]. In addition, the constant blood glucose testing and dose titration associated with insulin therapy are not necessary for exenatide treatment, which could lead to fewer incidences of hypoglycemia, making exenatide treatment simpler to administer and potentially improving treatment compliance and safety. Traditionally, when oral hypoglycemic agents have failed to properly control hyperglycemia, initiating insulin therapy has been the recommended next step in T2DM treatment. However, more and more guidelines have pointed out that GLP-1 agonists or SGLT2 inhibitors should be the first-line choice in patients whose blood glucose is not well controlled with oral 
Table 3 Summary of adverse events (AEs) (SS)

\begin{tabular}{|c|c|c|c|c|c|}
\hline \multirow[t]{2}{*}{ Adverse events (AEs) } & \multicolumn{2}{|c|}{ Exenatide $(N=52)$} & \multicolumn{2}{|c|}{$\begin{array}{l}\text { Biphasic insulin aspart } 30 \\
(N=52)\end{array}$} & \multirow[t]{2}{*}{$P$ value } \\
\hline & Events, $n$ & Patients, $n(\%)$ & Events, $n$ & Patients, $n(\%)$ & \\
\hline All AEs & 114 & $37(71.15)$ & 105 & $36(69.23)$ & 1 \\
\hline Study drug-related AEs ${ }^{\mathrm{a}}$ & 71 & $27(51.92)$ & 56 & $25(48.08)$ & 0.8446 \\
\hline AEs unrelated to the study drug & 43 & $23(44.23)$ & 49 & $25(48 / 08)$ & 0.8442 \\
\hline Severe AEs & 5 & $4(7.69)$ & 5 & $3(5.77)$ & 1 \\
\hline \multicolumn{6}{|l|}{ Study drug-related AEs ${ }^{\mathrm{a}}$} \\
\hline Dysglycemia $^{\mathrm{b}} /$ hypoglycemia & $20 / 17$ & $13(25.00) / 10(19.23)$ & $45 / 45$ & $19(36.54) / 19(36.54)$ & 0.2881 \\
\hline Headache/dizziness & 5 & $5(9.62)$ & 1 & $1(1.92)$ & 0.205 \\
\hline Abdominal discomfort & 3 & $3(5.77)$ & 0 & $0(0.00)$ & 0.2427 \\
\hline Dyslipidemia & 3 & $3(5.77)$ & 2 & $2(3.85)$ & 1 \\
\hline Decreased appetite & 3 & $2(3.85)$ & 0 & $0(0.00)$ & 0.4951 \\
\hline Upper respiratory tract & 2 & $2(3.85)$ & 0 & $0(0.00)$ & 0.4951 \\
\hline \multicolumn{6}{|l|}{ Infection } \\
\hline Constipation & 2 & $1(1.92)$ & 0 & $0(0.00)$ & 1 \\
\hline Allergic reactions to the drug & 2 & $1(1.92)$ & 0 & $0(0.00)$ & 1 \\
\hline Cardiac abnormalities & 1 & $1(1.92)$ & 0 & $0(0.00)$ & 1 \\
\hline Lack of attention/concentration & 1 & $1(1.92)$ & 0 & $0(0.00)$ & 1 \\
\hline Hip joint pain & 1 & $1(1.92)$ & 0 & $0(0.00)$ & 1 \\
\hline Abnormal liver function test results & 0 & $0(0.00)$ & 2 & $1(1.92)$ & 1 \\
\hline Urinary tract infection & 0 & $0(0.00)$ & 2 & $1(1.92)$ & 1 \\
\hline Abnormal hs-CRP level & 0 & $0(0.00)$ & 1 & $1(1.92)$ & 1 \\
\hline Skin allergies & 0 & $0(0.00)$ & 1 & $1(1.92)$ & 1 \\
\hline Upper/lower extremity pain & 0 & $0(0.00)$ & 1 & $1(1.92)$ & 1 \\
\hline Left heel pain & 0 & $0(0.00)$ & 1 & $1(1.92)$ & 1 \\
\hline
\end{tabular}

Data were presented as $n(\%)$ in patients who received $\geq 1$ dose of study drug and had baseline safety data and also safety data for at least 1 follow-up visit

$S S$ safety set, $h s-C R P$ high sensitivity $\mathrm{C}$-reactive protein

a Study drug-related AEs referred to AEs definitely or possibly related to the study drug or AEs possibly unrelated to the study drug

b Dysglycemia included hypoglycemia and other forms of dysglycemia 
hypoglycemic drugs $[17,18]$. Our findings suggest that exenatide could provide more benefits than insulin, especially in patients with obesity and T2DM who suffer from large postprandial glucose excursions.

Our study is limited by its open-label design, as the necessity of insulin dose adjustment during the study made a double-blinded design unfeasible. However, as investigators were blinded to the main efficacy parameters during the study, the subjective bias associated with an open-label study was minimized. In addition, since we ran into some unexpected difficulties in recruiting patients for our study, to prevent potentially endless delay in the study, we decided to conduct the study on the 104 participants we had already enrolled, and as a result, our sample size was smaller than the originally planned 150 participants. We acknowledge this as a major limitation in our study and consider it to be a possible factor underlying our results that exenatide did not significantly change major GV indices such as MAGE, LAGE, MODD, and SDBG.

\section{CONCLUSIONS}

In conclusion, exenatide showed a similar GV change, HbA1c reduction, decrease in inflammatory factors, and safety profile to BIAsp 30 . Moreover, exenatide was more effective in achieving control of PPG excursion and body weight than BIAsp 30. The dose of exenatide does not need to be titrated to blood glucose levels, so it could be a more convenient alternative treatment to BIAsp30, especially for patients with obesity and large PPG excursion.

\section{ACKNOWLEDGEMENTS}

We thank all the physicians and participants in the study for their cooperation and generous participation and assistance.

Funding. This study was sponsored by AstraZeneca China and 3SBio Inc. (study code: ESR-14-10319) and was supported by Multi- factorial Integrative Management of Type 2 Diabetes (MiDiab) of the Nation Key Research and Development Program of China (grant no. 2017YFC1309803) and the Key Research and Development Program of Shaanxi Province, China (grant no. 2017ZDCXLSF0201). The journal's Rapid Service Fee was funded by AstraZeneca China and 3SBio Inc.

Editorial Assistance. We thank Accdon (www.accdon.com) for its linguistic assistance during the preparation of this manuscript. This assistance was funded by AstraZeneca China and 3SBio Inc.

Authorship. All named authors meet the International Committee of Medical Journal Editors (ICMJE) criteria for authorship for this article, take responsibility for the integrity of the work as a whole, and have given their approval for this version to be published.

Authorship Contributions. Li Wang, Xiangyang Liu and Qiuhe Ji conceived of and designed the study; Li Wang, Xiangyang Liu, Jie Ming, Jingbo Lai, and Qiuhe Ji collected or assembled the data; Li Wang, Xiangyang Liu, Wenjuan Yang, Jingbo Lai, Xinwen Yu, Xiling Gao, Kaiyan Ma, Jing Xu, Zhufang Tian, Qingzhen $\mathrm{He}$, and Qiuhe Jian analyzed or interpreted the data; Li Wang and Xiangyang Liu wrote the first draft; Li Wang, Xiangyang Liu, and Qiuhe Ji contributed to the discussion and reviewed and edited the article. Li Wang and Xiangyang Liu contributed equally to this work.

Disclosures. Qiuhe Ji has attended advisory boards and been a speaker for Eli Lilly, Novo Nordisk, Merck Sharp \& Dohme China, Sanofi Aventis, Huadong Pharmaceuticals Company, and Medtronic, and has received research grants from Novo Nordisk, Merck Sharp \& Dohme China, and AstraZeneca. Li Wang, Xiangyang Liu, Wenjuan Yang, Jingbo Lai, Xinwen Yu, Jianrong Liu, Xiling Gao, Jie Ming, Kaiyan Ma, Jing $\mathrm{Xu}$, Zhufang Tian and Qingzhen He have nothing to disclose. 
Compliance with Ethics Guidelines. This trial was approved by the Independent Ethics Committee of Xijing Hospital, First Affiliated Hospital of Fourth Military Medical University (ID: KY20150319, KY201505051) (Xi'an, China), the Second Affiliated Hospital of Xi'an Jiaotong University (Xi'an, China), Chang An Hospital (Xi'an, China), Xi'an Gaoxin Hospital (Xi'an, China), Yan'an People's Hospital (Yan'an), China and Shangluo Central Hospital (Shangluo, China), and written informed consent was obtained from all participants. This trial was performed as per the ethics delineated in the Helsinki Declaration. This study is registered with ClinicalTrials.gov: NCT02449603.

Data Availability. The datasets generated during and/or analyzed during the current study are available from the corresponding author on reasonable request.

Open Access. This article is licensed under a Creative Commons Attribution-NonCommercial 4.0 International License, which permits any non-commercial use, sharing, adaptation, distribution and reproduction in any medium or format, as long as you give appropriate credit to the original author(s) and the source, provide a link to the Creative Commons licence, and indicate if changes were made. The images or other third party material in this article are included in the article's Creative Commons licence, unless indicated otherwise in a credit line to the material. If material is not included in the article's Creative Commons licence and your intended use is not permitted by statutory regulation or exceeds the permitted use, you will need to obtain permission directly from the copyright holder. To view a copy of this licence, visit http:// creativecommons.org/licenses/by-nc/4.0/.

\section{REFERENCES}

1. Xu S, Liu X, Ming J, Ji Q. Comparison of exenatide with biphasic insulin aspart 30 on glucose variability in type 2 diabetes: study protocol for a randomized controlled trial. Trials. 2016;24(17):160.
2. Hermansen $\mathrm{K}$, Colombo M, Storgaard H, Østergaard A, Kolendorf K, Madsbad S. Improved postprandial glycemic control with biphasic insulin aspart relative to biphasic insulin lispro and biphasic human insulin in patients with type 2 diabetes. Diabetes Care. 2002;25(5):883-8.

3. Liebl A, Mohan V, Yang W, Strojek K, Linjawi S. 15 years of experience with biphasic insulin aspart 30 in type 2 diabetes. Drugs R\&D. 2018;18(1):27-39.

4. Gao Y, Wang K, Chen Y, Shen L, Hou J, Xuan J, et al. A real-world anti-diabetes medication cost comparison between premixed insulin analogs and long-acting insulin analogs in chinese patients with type 2 diabetes: a retrospective database analysis. Diabetes Ther. 2018;9(2):673-82.

5. Yang W, Ersoy C, Wang G, Ye S, Liu J, Miao H, et al. Efficacy and safety of three-times-daily versus twicedaily biphasic insulin aspart 30 in patients with type 2 diabetes mellitus inadequately controlled with basal insulin combined with oral antidiabetic drugs. Diabetes Res Clin Pract. 2019;150:158-66.

6. Ligthelm RJ. Self-titration of biphasic insulin aspart 30/70 improves glycaemic control and allows easy intensification in a Dutch clinical practice. Primary Care Diabetes. 2009;3(2):97-102.

7. Farcasiu E, Ivanyi T, Mozejko-Pastewka B, Birkus Z, Csog J, Kowalska I, et al. Efficacy and safety of prandial premixed therapy using insulin lispro mix 50/50 3 times daily compared with progressive titration of insulin lispro mix $75 / 25$ or biphasic insulin aspart 70/30 twice daily in patients with type 2 diabetes mellitus: a randomized, 16-week, open-label study. Clin Ther. 2011;33(11):1682-93.

8. Briones M, Bajaj M. Exenatide: a GLP-1 receptor agonist as novel therapy for Type 2 diabetes mellitus. Expert Opin Pharmacother. 2006;7(8):1055-64.

9. Washington MC, Raboin SJ, Thompson W, Larsen CJ, Sayegh AI. Exenatide reduces food intake and activates the enteric nervous system of the gastrointestinal tract and the dorsal vagal complex of the hindbrain in the rat by a GLP-1 receptor. Brain Res. 2010;16(1344):124-33.

10. Egan JM, Clocquet AR, Elahi D. The insulinotropic effect of acute exendin- 4 administered to humans: comparison of nondiabetic state to type 2 diabetes. J Clin Endocrinol Metab. 2002;87(3):1282-90.

11. Sennik D, Ahmed F, Russell-Jones D. Exenatide, a GLP-1 agonist in the treatment of Type 2 diabetes. Expert Rev Endocrinol Metab. 2012;7(1):15-26.

12. Bradley DP, Kulstad R, Schoeller DA. Exenatide and weight loss. Nutrition. 2010;26(3):243-9. 
13. DeFronzo RA, Ratner RE, Han J, Kim DD, Fineman MS, Baron AD. Effects of exenatide (exendin-4) on glycemic control and weight over 30 weeks in metformin-treated patients with type 2 diabetes. Diabetes Care. 2005;28(5):1092-100.

14. Morgan CL, Qiao Q, Grandy S, Johnsson K, JenkinsJones S, Holden S, et al. Glucose control and weight change associated with treatment with exenatide compared with basal insulin: a retrospective study. Diabetes Ther. 2018;9(1):269-83.

15. Levin PA, Nguyen H, Wittbrodt ET, Kim SC. Glucagon-like peptide- 1 receptor agonists: a systematic review of comparative effectiveness research. Diabetes Metab Syndr Obes Targ Ther. 2017;10:123-39.

16. Abdul-Ghani MA, Puckett C, Triplitt C, Maggs D, Adams J, Cersosimo $\mathrm{E}$, et al. Initial combination therapy with metformin, pioglitazone and exenatide is more effective than sequential add-on therapy in subjects with new-onset diabetes. Results from the Efficacy and Durability of Initial Combination Therapy for Type 2 Diabetes (EDICT): a randomized trial. Diabetes Obes Metab. 2015;17(3): 268-75.

17. Cosentino F, Grant PJ, Aboyans V, Bailey CJ, Ceriello A, Delgado V, et al. 2019 ESC Guidelines on diabetes, pre-diabetes, and cardiovascular diseases developed in collaboration with the EASD. Eur Heart J. 2020;41(2):255-32323.

18. American DA. Diabetes advocacy: standards of medical care in diabetes-2019. Diabetes Care. 2020;43(Suppl 1):S1-S204.

19. Nauck MA, Duran S, Kim D, Johns D, Northrup J, Festa A, et al. A comparison of twice-daily exenatide and biphasic insulin aspart in patients with type 2 diabetes who were suboptimally controlled with sulfonylurea and metformin: a non-inferiority study. Diabetologia. 2007;50(2):259-67.

20. Blonde L. Current antihyperglycemic treatment strategies for patients with type 2 diabetes mellitus. Clevel Clin J Med. 2009;76(Suppl 5):S4-11.

21. Tang X, Li S, Wang Y, Wang M, Yin Q, Mu P, et al. Glycemic variability evaluated by continuous glucose monitoring system is associated with the $10-\mathrm{y}$ cardiovascular risk of diabetic patients with wellcontrolled HbA1c. Clin Chim Acta Int J Clin Chem. 2016;461:146-50.

22. Skrha J, Soupal J, Skrha J Jr, Prazny M. Glucose variability, HbA1c and microvascular complications. Rev Endocr Metab Disord. 2016;17(1): 103-10.

23. Cardoso CRL, Leite NC, Moram CBM, Salles GF. Long-term visit-to-visit glycemic variability as predictor of micro- and macrovascular complications in patients with type 2 diabetes: the Rio de Janeiro Type 2 Diabetes Cohort Study. Cardiovasc Diabetol. 2018;17(1):33.

24. Hirsch IB. Intensifying insulin therapy in patients with type 2 diabetes mellitus. Am J Med. 2005;118(Suppl 5A):21S-S2626.

25. Wu JD, Xu XH, Zhu J, Ding B, Du TX, Gao G, et al. Effect of exenatide on inflammatory and oxidative stress markers in patients with type 2 diabetes mellitus. Diabetes Technol Ther. 2011;13(2):143-8.

26. Liu X, Huang J, Li J, Mao Q, He J. Effects of liraglutide combined with insulin on oxidative stress and serum MCP-1 and NF-kB levels in type 2 diabetes. J Coll Phys Surg Pak. 2019;29(3):218-21.

27. Ceriello A, Esposito K, Piconi L, Ihnat MA, Thorpe $\mathrm{JE}$, Testa R, et al. Oscillating glucose is more deleterious to endothelial function and oxidative stress than mean glucose in normal and type 2 diabetic patients. Diabetes. 2008;57(5):1349-54.

28. Saisho Y. Glycemic variability and oxidative stress: a link between diabetes and cardiovascular disease? Int J Mol Sci. 2014;15(10):18381-406.

29. Nusca A, Tuccinardi D, Albano M, Cavallaro C, Ricottini E, Manfrini S, et al. Glycemic variability in the development of cardiovascular complications in diabetes. Diabetes Metab Res Rev. 2018;34(8): e3047.

30. Cavalot F. Do data in the literature indicate that glycaemic variability is a clinical problem? Glycaemic variability and vascular complications of diabetes. Diabetes Obes Metab. 2013;15(Suppl 2): 3-8.

31. Gallwitz B, Bohmer M, Segiet T, Molle A, Milek K, Becker B, et al. Exenatide twice daily versus premixed insulin aspart 70/30 in metformin-treated patients with type 2 diabetes: a randomized 26-week study on glycemic control and hypoglycemia. Diabetes Care. 2011;34(3):604-6.

32. Pawaskar MD, Blickensderfer AL, Hoogwerf BJ, Quimbo R, Wade R. Hypoglycemia in patients with type 2 diabetes using concomitant exenatide BID and long-acting insulin therapy. J Med Econ. 2011;14(6):705-8.

33. Fabunmi R, Nielsen LL, Quimbo R, Schroeder B, Misurski D, Wintle M, et al. Patient characteristics, drug adherence patterns, and hypoglycemia costs for patients with type 2 diabetes mellitus newly initiated on exenatide or insulin glargine. Curr Med Res Opin. 2009;25(3):777-86. 
34. Irace C, Fiorentino R, Carallo C, Scavelli F, Gnasso A. Exenatide improves glycemic variability assessed by continuous glucose monitoring in subjects with type 2 diabetes. Diabetes Technol Ther. 2011;13(12):1261-3.

35. McCall AL, Cox DJ, Brodows R, Crean J, Johns D, Kovatchev B. Reduced daily risk of glycemic variability: comparison of exenatide with insulin glargine. Diabetes Technol Ther. 2009;11(6):339-44.

36. Yin TT, Bi Y, Li P, Shen SM, Xiong XL, Gao LJ, et al. Comparison of glycemic variability in Chinese T2DM patients treated with exenatide or insulin glargine: a randomized controlled trial. Diabetes Ther. 2018;9(3):1253-67.

37. Joseph JI, Torjman MC, Strasma PJ. Vascular glucose sensor symposium: continuous glucose monitoring systems (CGMS) for hospitalized and ambulatory patients at risk for hyperglycemia, hypoglycemia, and glycemic variability. J Diabetes Sci Technol. 2015;9(4):725-38.

38. Tsujino D, Nishimura R, Onda Y, Seo C, Ando K, Utsunomiya K. Biphasic insulin aspart-30 reduces glycemic variability to a greater degree than insulin detemir: A randomized controlled trial of oncedaily insulin regimens using continuous glucose monitoring. J Diabetes Investig. 2017;9:573-8.

39. Ohta A, Suwa T, Sada Y, Kato H, Koganei R, Asai S, et al. Comparison of daily glucose excursion by continuous glucose monitoring between type 2 diabetic patients receiving biphasic insulin aspart 30 or biphasic human insulin 30. J Diabetes Investig. 2011;2(5):406-11.

40. Zheng F, Lu W, Jia C, Li H, Wang Z, Jia W. Relationships between glucose excursion and the activation of oxidative stress in patients with newly diagnosed type 2 diabetes or impaired glucose regulation. Endocrine. 2010;37(1):201-8.
41. Nakanishi S, Yoneda M, Maeda S. Impact of glucose excursion and mean glucose concentration in oral glucose-tolerance test on oxidative stress among Japanese Americans. Diabetes Metab Syndr Obes Targ Ther. 2013;6:427-33.

42. Investigators F-ST. Glucose variability in a 26-week randomized comparison of mealtime treatment with rapid-acting insulin versus GLP-1 Agonist in participants with type 2 Diabetes at high cardiovascular risk. Diabetes Care. 2016;39(6):973-81.

43. Gentilella R, Bianchi C, Rossi A, Rotella CM. Exenatide: a review from pharmacology to clinical practice. Diabetes Obes Metab. 2009;11(6):544-56.

44. McBride PE, Einerson JA, Grant $H$, Sargent C, Underbakke G, Vitcenda M, et al. Putting the Diabetes Prevention Program into practice: a program for weight loss and cardiovascular risk reduction for patients with metabolic syndrome or type 2 diabetes mellitus. J Nutr Health Aging. 2008;12(10): 745S-S749749.

45. Homan EA, Reyes MV, Hickey KT, Morrow JP. Clinical overview of obesity and diabetes mellitus as risk factors for atrial fibrillation and sudden cardiac death. Front Physiol. 2018;9:1847.

46. Arnolds S, Dellweg S, Clair J, Dain MP, Nauck MA, Rave K, et al. Further improvement in postprandial glucose control with addition of exenatide or sitagliptin to combination therapy with insulin glargine and metformin: a proof-of-concept study. Diabetes Care. 2010;33(7):1509-15.

47. Ludwig S, Shen GX. Statins for diabetic cardiovascular complications. Curr Vasc Pharmacol. 2006;4(3):245-51. 